\title{
Conocimientos, actitudes y prácticas sobre toxoplasmosis en dos comunas de Armenia, Quindío, con alta prevalencia de la infección
}

\author{
Knowledge, attitudes, and practices related to toxoplasmosis in two districts of \\ high prevalence in Armenia, Quindío
}

\section{Conhecimentos, atitudes e práticas sobre toxoplasmose em duas comunas de Armenia, Quindío, com alta prevalência de infecção}

Daniel Celis-Giraldo"; Laura Lorena García López²; Juan David Valencia Hernández³; John Alejandro Acosta Dávila4; Mónica Vargas Montes ${ }^{5}$; Jorge Enrique Gómez Marín ${ }^{6}$

1 Médico. Universidad del Quindío, Armenia, Colombia. dcelisg_1@uqvirtual.edu.co. orcid: https://orcid.org/0000-0002-8428-2740

2 Magíster en Ciencias Biomédicas. Universidad del Quindío, Armenia, Colombia. Ilgarcial@uqvirtual.edu.co. orcid: https://orcid. org/0000-0002-9061-2830

3 Biólogo. Universidad del Quindío. Armenia, Colombia. jdvalenciah@uqvirtual.edu.co. orcid: https://orcid.org/0000-0002-9189-5684

4 Doctor en Ciencias Biomédicas. Universidad del Quindío, Armenia, Colombia. jaacostad@uqvirtual.edu.co. orcid: https://orcid. org/0000-0003-1407-1232

5 Magíster en Ciencias Biomédicas. Universidad del Quindío, Armenia, Colombia. mvargasm@uqvirtual.edu.co. orcid: https://orcid. org/0000-0003-2418-3596

6 Médico, doctor (PhD) en Biología Parasitaria. Universidad del Quindío, Armenia, Colombia. gepamol2@uniquindio.edu.co. orcid: https://orcid.org/0000-0001-6472-3329

Recibido: 03/12/2020. Aprobado: 09/11/2021. Publicado: 06/12/2021

Celis-Giraldo D, García-López LL, Valencia-Hernández JD, Acosta-Dávila JA, Vargas-Montes M, Gómez-Marín JE. Conocimientos, actitudes y prácticas sobre toxoplasmosis en dos comunas de Armenia, Quindío, con alta prevalencia de la infección. Rev. Fac. Nac. Salud Pública. 2022; 40(1):e344576. Dor: https://doi.org/10.17533/udea.rfnsp.e344576

\section{Resumen}

Objetivo: Describir los conocimientos, las actitudes y las prácticas acerca de la toxoplasmosis en dos comunas de Armenia, Quindío, con alta prevalencia de la infección. Metodología: Estudio descriptivo con población de dos comunas de Armenia, Quindío. Se aplicó un cuestionario autodiligenciado tipo conocimientos, actitudes y prácticas.
Esta herramienta incluyó elementos sobre el parásito Toxoplasma gondii, sus vías de transmisión, aspectos clínicos, diagnósticos y de tratamiento generales, así como prácticas para evitar la infección. El instrumento se aplicó antes y 
después de una intervención educativa. Se describieron las frecuencias en el número de respuestas correctas antes y después de la intervención para cada comuna. Resultados: Participaron 27 personas, con una media de edad de 57 años. El 59 \% fueron mujeres. El 48\% había completado la educación media y el 40,7 \% la primaria. El conocimiento del agente causal antes de la intervención fue del $22 \%$, mientras que posterior a la intervención fue del 92,3\% en la comuna 1 y del $81,8 \%$ en la comuna 6 . Posterior a la intervención, cerca del $90 \%$ de los encuestados reconoció la retina como la principal estructura afectada y todos los encuestados reconocieron el consumo de agua hervida como factor protector. Conclusión: Los conocimientos sobre la toxoplasmosis en las dos comunas eran limitados. Luego de la intervención educativa, se evidenció un aumento en el porcentaje de respuestas correctas en la mayoría de las preguntas. Se recomienda realizar nuevas intervenciones educativas y en salud pública, para evaluar los efectos de estas a largo plazo.

--------Palabras clave: Conocimientos, actitudes y prácticas en salud, factores de riesgo, toxoplasmosis, Armenia (Quindío).

\section{Abstract}

Objective: To describe knowledge, attitudes and practices related to toxoplasmosis in two districts of high prevalence in Armenia, Quindío. Methodology: descriptive study; the population of two districts of Armenia, Quindío were engaged. A self-administered questionnaire regarding knowledge, attitudes, and practices was applied. This tool included elements related to Toxoplasma gondii, its transmission pathways, general clinical, diagnostic and treatment aspects, as well as practices to prevent infection. The instrument was applied before and after an educational intervention. Frequencies were described as the number of correct answers before and after the intervention for each district. Results: 27 people participated, with an average age of 57 years. $59 \%$ were women; $48 \%$ had completed high school and $40.7 \%$ had completed elementary school. Before the intervention, the knowledge of the causal agent was $22 \%$, while after the intervention, it was $92.3 \%$ in district 1 and $81.8 \%$ in district 6 . After the intervention, about $90 \%$ of participants recognized the retina as the main compromised structure and all participants recognized the consumption of boiled water as a protective factor. Conclusion: The knowledge regarding toxoplasmosis in the two districts was limited. After the educational intervention, there was an increase in the percentage of correct answers in most of the questions. New educational and public health interventions are recommended to assess the long-term effects of these interventions.

---------Keywords: Knowledge, attitudes and practices in health, risk factors, toxoplasmosis, Armenia (Quindío).

\section{Resumo}

Objetivos: O objetivo deste estudo é descrever os problemas, as ações e as práticas sobre a toxoplasmose nas comunidades da Armênia, Quindío, onde se nota alta prevalência da infecção. Metodologia: Este é um estudo descritivo que abrange uma população de duas comunas na Armênia, Quindío. Foi implementada uma ferramenta, que consiste em questionários destinados àquela população e aplicáveis pelos seus próprios membros, que correspondem a conhecimentos, atitudes e práticas. Essa ferramenta inclui elementos sobre o parasito Toxoplasma gondii, suas vias de transmissão, aspectos clínicos gerais, diagnósticos e tratamento, e práticas de prevenção. $\mathrm{O}$ instrumento foi aplicado antes e após uma intervenção educativa exata. Foram descritas as frequências do número de acertos, antes e depois da intervenção para cada comuna. Resultados: participaram 27 pessoas, com média de idade de 57 anos, das quais $59 \%$ eram mulheres e $48 \%$ tinham ensino médio completo e 40,7 \% ensino primário. O conhecimento do agente causal antes da intervenção havia em $22 \%$, enquanto que após a intervenção, passou para $92,3 \%$ na comuna 1 , e $81,8 \%$ na comuna 6. Após a intervenção, cerca de $90 \%$ dos entrevistados reconheceram a retina como a estrutura mais afetada e todos os entrevistados reconheceram que o consumo de água fervida é um fator de proteção. Conclusão: Desconhecimento sobre a toxoplasmose nas duas comunas. Após a intervenção educativa, houve evidência de aumento do percentual de acertos na maioria das questões. Recomenda-se a realização de novas intervenções educacionais e de saúde pública, para avaliar os efeitos destas a longo prazo na populacão.

------Palavras-chave: Conhecimentos, Atitudes e Prática em Saúde, Fatores de Risco, Toxoplasmosis, Armenia (Quindío). 


\section{Introducción}

La toxoplasmosis es una enfermedad transmitida por alimentos, agua y contacto con felinos que excretan activamente ooquistes a través de sus heces, y se considera una infección de preocupación global [1]. "Es producida por el parásito intracelular obligado Toxoplasma gondii" [2]."

"El parásito infecta un amplio espectro de huéspedes vertebrados, incluyendo al humano" [3]. "Su ciclo sexuado se da en el intestino de los felinos, que son sus hospederos" [2]. Los hospederos intermediarios, entre ellos el humano, se infectan mediante la ingestión de agua, verduras y frutas contaminadas con ooquistes viables, esporulados después de su eliminación previa en las heces de los felinos [4-6]; también a través de la carne poco cocida que contiene quistes tisulares del parásito. Este también puede atravesar la placenta, en el caso de la transmisión materno-fetal [7-9].

Las manifestaciones clínicas de la toxoplasmosis son amplias, desde un síndrome de mononucleosis infecciosa o un cuadro similar a neumonía viral, hasta el desarrollo de encefalitis, miocarditis y retinocoroiditis. El ojo es el principal órgano afectado en el paciente inmunocompetente $y$, en general, se evidencian cicatrices al fondo de ojo en cerca del $10 \%$ de las personas infectadas [10-12].

La prevalencia de la toxoplasmosis varía entre el 20 y el $60 \%$, dependiendo de la ubicación geográfica $[9,13,14]$, y la "frecuencia y severidad tienen relación inversa con la latitud" [15]. En América Latina, la presentación clínica es más severa que en países como Estados Unidos y Francia, en parte por la mayor frecuencia de cepas virulentas que circulan en Latinoamérica $[9,16-$ 19]. En Colombia, se ha reportado seropositividad, lo cual indica contacto previo con el parásito, "en cerca del $50 \%$ de la población" [20]. De acuerdo con ello, se espera que por lo menos 2,5 millones de colombianos tendrían compromiso ocular con cicatrices en la retina por toxoplasmosis, que llevarían a por lo menos en el $20 \%$ (cerca de 400.000 personas) a ceguera legal en el ojo afectado [21].

En Quindío, departamento de Colombia, la presentación de la enfermedad es particular, por la alta prevalencia de la infección y el número de casos severos reportados. Además, se ha evidenciado la presencia del ácido desoxirribonucleico $(\mathrm{ADN})$ del parásito en carne y fuentes de agua de consumo humano en la región [22,23], así como en restaurantes escolares [24]. La calidad de vida también es un aspecto que se ha visto afectado en pacientes con toxoplasmosis ocular, generando una grave afectación en quienes la padecen $[25,26]$. Nuestro grupo

\footnotetext{
Todas las traducciones son de los autores.
}

de investigación GEPAMOL ha evaluado que las comunas 1 y 6 de Armenia tienen una prevalencia elevada de anticuerpos IgG anti-Toxoplasma en niños, siendo del $70 \mathrm{y}$ del $36,5 \%$ respectivamente [24]. Por lo tanto, hay evidencia de que se presenta una exposición temprana a la infección en estas comunidades, con riesgo de desarrollar secuelas durante el resto de la vida. En el año 2019, en estas dos comunas documentamos la prevalencia de la toxoplasmosis en población general, siendo esta del $69,5 \%$, de los cuales el 15,1\% presentaba lesiones de retinocoroiditis al fondo de ojo [27].

La educación en salud es vital para el empoderamiento de las comunidades y a la hora de formular y evaluar programas de educación cuyo objetivo es prevenir la enfermedad y poder priorizar las pautas que aún no se han tenido en cuenta en las poblaciones de riesgo, de tal manera que las personas se apropien del conocimiento en salud y mejoren sus hábitos de vida para la prevención de la enfermedad [28].

En el caso de la toxoplasmosis humana, es de suma importancia conocer las formas de transmisión de la enfermedad, para disminuir la probabilidad de adquirir la infección [29,30]. En un ensayo clínico en embarazadas de Canadá, los autores concluyeron que "la educación prenatal puede cambiar de forma efectiva el comportamiento de las embarazadas, ya que aumenta la higiene doméstica, personal y de los alimentos" [31, p. 494]. Otro estudio en Polonia confirmó que "el 89,7\% (26 de 29) las madres de recién nacidos infectados practicaban comer carne cruda o tenían un contacto cercano con gatos pequeños" [32, p. 542]. La necesidad de una mejor educación sanitaria en los jóvenes, en los que la seropositividad a Toxoplasma y las tasas de embarazo precoz son relativamente altas, está bien fundamentada [32].

En el Quindío no se cuenta con estudios previos que evalúen los conocimientos, las actitudes y las prácticas (CAP) sobre toxoplasmosis ni se han documentado estrategias de educación y apropiación social del conocimiento en salud que puedan ayudar a prevenir la infección en la población general. Por lo tanto, el presente estudio tuvo como objetivo describir los conocimientos, las actitudes y las prácticas acerca de la toxoplasmosis en dos comunas de Armenia, Quindío, con alta prevalencia de la infección, por medio de la aplicación de encuestas pre y postintervención.

\section{Metodología}

A continuación se presenta el tipo de estudio realizado, la población y muestra definidas para el mismo, la descripción de los cuestionarios tipo CAP pre y postintervención, las actividades educativas desarrolladas y el análisis de la información. 
El estudio realizado fue de tipo descriptivo, el cual consiste en la observación de eventos presentes en una población de estudio, y muestra la frecuencia y las características con las que se presenta una enfermedad en un momento determinado. La recolección de datos se hace en una sola ocasión y de inmediato se procede a su descripción y análisis [33].

\section{Población y muestra}

El estudio valoró los CAP que tenían las personas de dos comunas (Comuna 1 - "Centenario", y Comuna 6 - "San José") de la ciudad de Armenia, Quindío, que asistieron a un taller comunitario de educación en salud en marzo de 2020 (véase Figura 1). Las poblaciones fueron seleccionadas con base en los resultados de un tamizaje de salud visual realizado en junio del 2019 en ambas comunas, donde se encontró una prevalencia de anticuerpos $\operatorname{IgG}$ anti-Toxoplasma del $75 \%$ para la comuna $1(51 / 68)$ y del $65,5 \%$ para la comuna $6(61 / 93)$, con afectación ocular en el $16,6 \%(10 / 51)$ y el $11,4 \%(7 / 61)$ respectivamente [28].

La población fue convocada a través de medios de difusión local y puerta a puerta días antes de la actividad. Se incluyeron personas mayores de edad que qui- sieran participar de la actividad. Se excluyeron encuestas con datos insuficientes para efectuar el análisis de la información.

\section{Cuestionario y actividades de educación comunitaria}

Se construyó y aplicó un cuestionario tipo CAP. La encuesta fue estructurada y diseñada para ser autodiligenciada de forma privada por cada participante. Se realizó una encuesta previa a la intervención de educación comunitaria, para establecer una base de los conocimientos y las percepciones de la comunidad sobre la toxoplasmosis.

Luego de la encuesta preliminar, se dieron tres charlas educativas de 10 minutos, acerca del parásito, los medios de transmisión de la infección y la anatomía del ojo. Posteriormente, se llevó a cabo una actividad práctica, donde los participantes construyeron un modelo del ojo humano e identificaron la anatomía e importancia de cada estructura. Asimismo, se realizó un diálogo comunitario con preguntas, respuestas y contrapreguntas. Finalmente, se aplicó un cuestionario para evaluar si las personas aumentaron sus conocimientos y modificaron algunas actitudes sobre diferentes aspectos de la enfermedad.

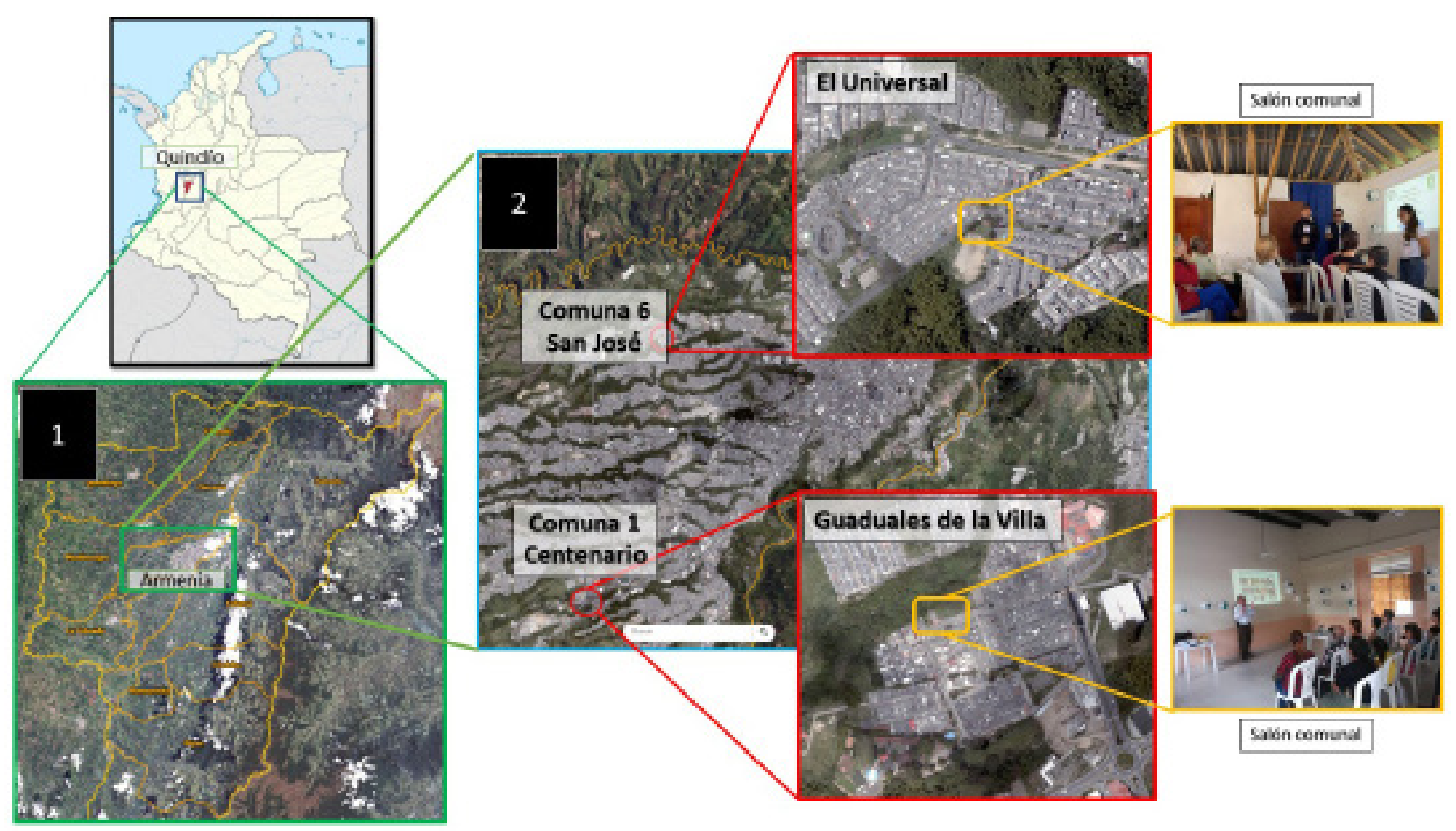

Figura 1. Distribución geográfica de los sitios de intervención comunitaria en dos comunas de Armenia, Quindío. En el departamento de Quindío, (1) se ubica la ciudad de Armenia, su capital. En la zona urbana (2) se ubican las comunas 1 y 6, y los barrios de encuentro para la intervención de educación en salud (El Universal y Guaduales de la Villa), así como el contexto de las actividades desarrolladas en el salón comunal de cada barrio en marzo de 2020.

Fuente: Elaboración propia a partir de [34]. 
Las preguntas fueron dicotómicas y de selección múltiple. En la clasificación dicotómica, se asumió que el encuestado poseía conocimiento si contestaba correctamente algún elemento de la enfermedad, como, por ejemplo, un síntoma, un vector, medidas de prevención, etc., sin evaluar en forma diferencial qué aspecto se trataba. Las preguntas múltiples tenían una sola respuesta, que se calificó como correcta siempre y cuando lo fuese, y evaluaba el grado de certeza del encuestado.

Se preguntó sobre el agente causal, la forma de transmisión, el vector u hospedero definitivo, las manifestaciones clínicas en el humano, el diagnóstico y el tratamiento. También acerca de las creencias y actitudes que tenían las personas sobre estos aspectos y si realizaban o no prácticas que aumentaban o prevenían el riesgo de contraer la enfermedad.

La encuesta preintervención contó con 14 preguntas, y la encuesta postintervención, con 11 . No se hicieron preguntas sobre prácticas postintervención, ya que se requiere un período más amplio, en donde se pueda evaluar y evidenciar una modificación clara en las prácticas de dicha población.

Los datos obtenidos con base al instrumento de recolección de información fueron revisados, asegurando que el porcentaje de las respuestas para todas las variables fueran mayor que el $95 \%$. Las preguntas abiertas fueron categorizadas posteriormente según el tipo de respuesta.

\section{Análisis estadístico}

La base de datos se digitalizó en Excel@ y se procesó con el software Epi-Info versión 7.2 (Centro de Control de Enfermedades, Atlanta, USA).

Se utilizaron medidas de tendencia central y de dispersión. Se comparó la diferencia de proporciones de las respuestas correctas antes y después de la intervención educativa.

\section{Aspectos bioéticos}

Todas las personas tuvieron la libertad de decidir su participación en el estudio, con el diligenciamiento previo del consentimiento informado por escrito.

Se les garantizó el anonimato y la confidencialidad de sus respuestas, con base en la Declaración de Helsinki [35] y la Resolución 8430 de 1993 [36] sobre la investigación en seres humanos.

\section{Resultados}

Se presentan, en primera instancia, los resultados de las encuestas de preintervención, respecto a los conocimientos sobre la transmisión, causa y síntomas de la toxoplasmosis, sobre su diagnóstico y tratamiento, las actitudes de riesgo para la transmisión y búsqueda de atención médica, así como las prácticas sobre prevención de la infección. Posteriormente, se muestran los resultados obtenidos de las encuestas postintervención, una vez realizadas las charlas educativas y el taller antes descrito en la metodología.

\section{Encuestas preintervención}

Se aplicaron un total de 27 encuestas preintervención. El 59,3 \% (16/27) de los encuestados fueron del sexo femenino. La mediana de edad fue de 57 años (rango intercuartil — RIC-: 53-73). El nivel educativo fue, en su mayoría, educación media y educación básica primaria, con un $48,1 \%(13 / 27)$ y $40,7 \%(11 / 27)$ respectivamente. Solo el 3,7 \% (1/27) de los encuestados tenía educación universitaria (véase Tabla 1).

Tabla 1. Características sociodemográficas de dos comunas en Armenia, Quindío, con alta prevalencia de toxoplasmosis.

\begin{tabular}{lcc}
\hline \multicolumn{1}{c}{ Variables } & \multicolumn{2}{c}{ Preintervención $(\mathbf{n}=\mathbf{2 7})$} \\
\hline Sociodemográficas & Comuna $1(\mathrm{n}=13)$ & Comuna $6(\mathrm{n}=14)$ \\
Edad (años) & $57(28-87)$ & $60(12-86)$ \\
Sexo (\%) $\quad$ Masculino & $23(3 / 13)$ & $21,5(3 / 14)$ \\
\multicolumn{1}{c}{ Femenino } & $77(10 / 13)$ & $78,5(11 / 14)$ \\
Años de estudio & $5(0-16)$ & $9(5-11)$ \\
\hline
\end{tabular}

Conocimientos sobre transmisión-causa-sintomas de la toxoplasmosis

El 44,4 \% (12/27) de los entrevistados sostenía que la toxoplasmosis era causada por una bacteria, mientras que el 22,2 \% (6/27) lo asoció a un parásito. En cuanto al animal que podría transmitir la enfermedad, el 40,7 $\%(11 / 27)$ de las personas encuestadas relacionó al gato como el hospedero de la enfermedad. Los síntomas que consideraron los encuestados se pueden presentar fueron visión borrosa, moscas volantes y unas lucecitas en el ojo (véase Tabla 2).

\section{Conocimientos sobre diagnóstico-tratamiento previos}

La mayoría de los encuestados afirmó que el diagnóstico se realiza mediante el análisis de una muestra de sangre, 
Tabla 2. Conocimiento, actitudes y prácticas de dos comunas antes y después de una intervención educativa sobre toxoplasmosis en Armenia, Quindío.

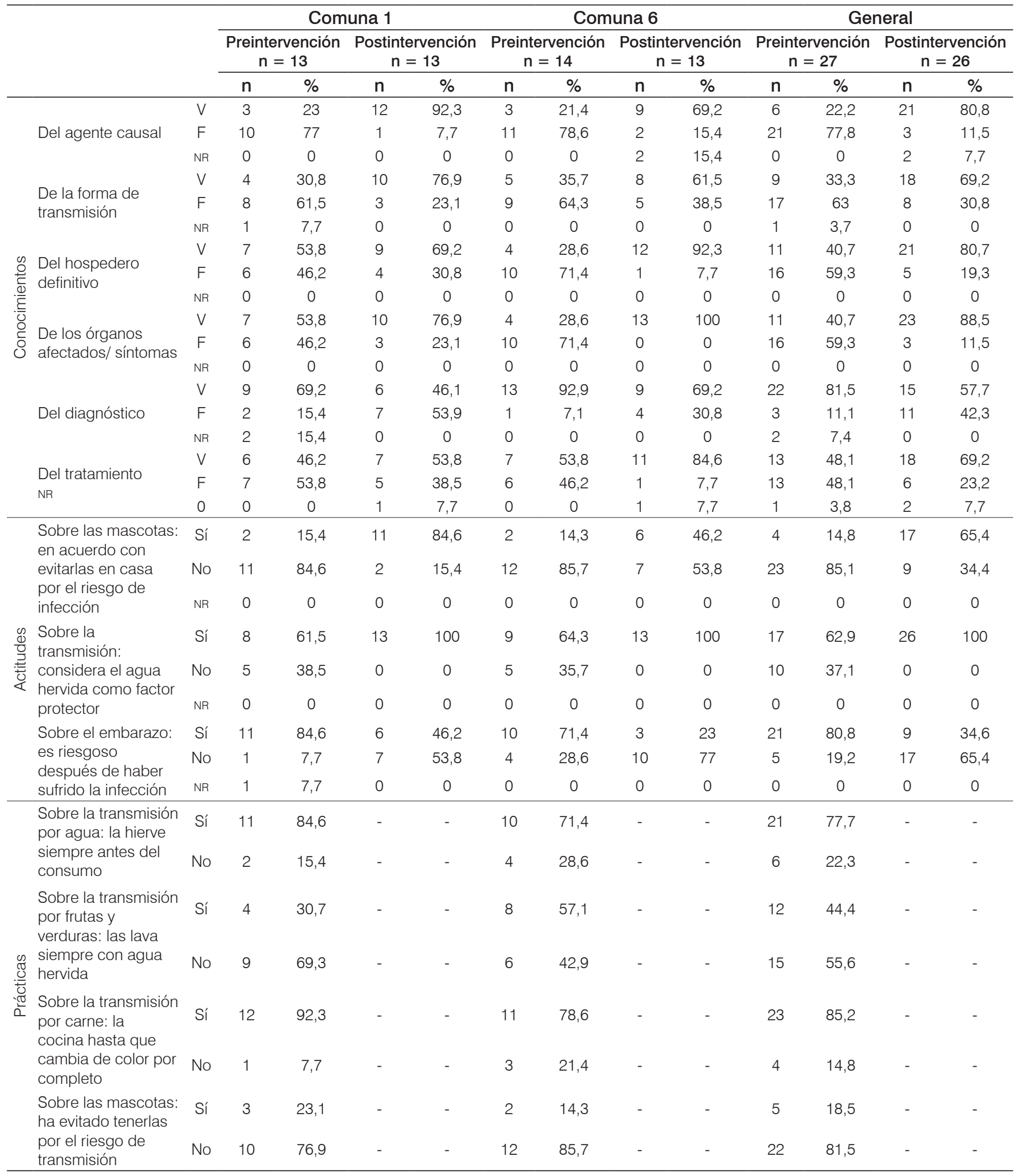

V: Verdadero; F: Falso; NR: No responde 
y el $50 \%(13 / 26)$ coincidió en que el tratamiento se hace con antibióticos y antiparasitarios.

Actitudes sobre riesgo de transmisión y búsqueda de atención médica

El 85,1 \% (23/27) de los encuestados no dejaría de tener una mascota en su hogar por el riesgo de contraer la enfermedad.

El 62,9 \% (17/27) consideró que si consumían agua hervida o de botella, esto ayudaría a prevenir la enfermedad.

El 77,7 \% (21/27) creía que era riesgoso tener un embarazo después de haber padecido la enfermedad.

\section{Prácticas sobre prevención}

El 77,7 \% (21/27) afirmó que hervía el agua antes del consumo.

En la manipulación de alimentos, el 55,6 \% (15/27) de las personas no lavaba las verduras con agua hervida, y el $85,2 \%(23 / 27)$ manifestaron cocer la carne por completo al consumirla.

\section{Encuestas postintervención}

Luego de realizar las charlas y el taller de manualidades, se practicaron las encuestas postintervención.

El 87,5\% (21/24) consideró a Toxoplasma gondii como el agente etiológico de la infección. El 69,2 \% $(18 / 26)$ afirmó que el agua era la principal fuente de infección. El 80,7 \% (21/26) señaló al gato como el hospedero definitivo.

El 57,7 \% (15/26) de las personas manifestaron que el diagnóstico se hace a partir de anticuerpos tomados de una muestra de sangre y el $72 \%(18 / 25)$ acertaron en que el tratamiento se debe llevar a cabo con antibióticos/ antiparasitarios y antiinflamatorios.

La Figura 2 muestra la distribución de la población antes y después de la intervención educativa según cada comuna. La comuna 1 presentó el 69,2 \% (9/13) de respuestas correctas en cuanto al conocimiento del hospedero definitivo, mientras que la comuna 6 contestó de manera acertada en el 92,3\% (12/13) de los encuestados. En la comuna 1 se observó que el porcentaje de

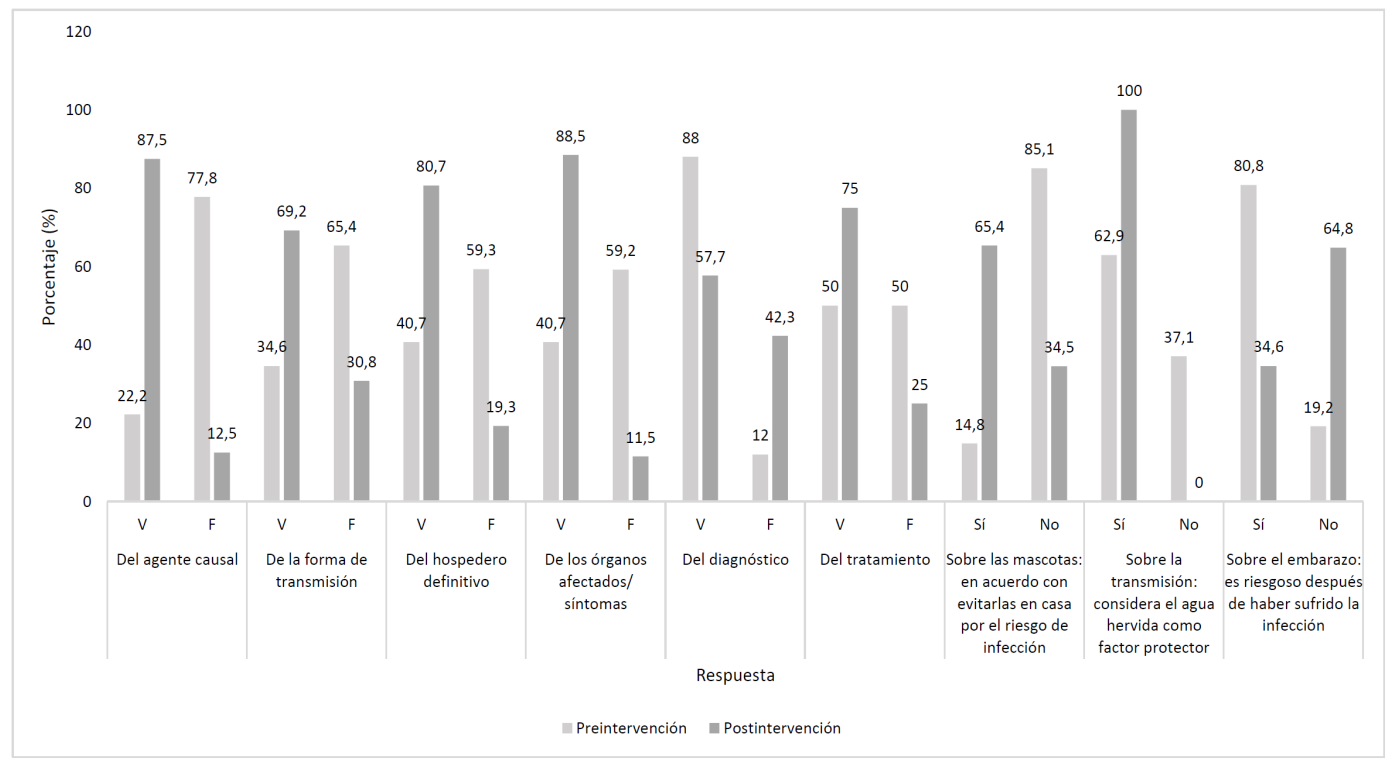

Figura 2. Distribución de conocimientos y actitudes sobre la toxoplasmosis antes y después de una intervención educativa en dos comunas de Armenia, Quindío

encuestados que estimaba riesgoso tener un embarazo tras haber tenido la infección previamente pasó del $91,7 \%(11 / 12)$ al $46,2 \%(6 / 13)$.

Sobre el agente causal, el porcentaje de respuestas acertadas fue significativamente mayor en cada comuna, con un $92,3 \%$ (comuna 1 ) y un $81,8 \%$ (comuna 6 ) de respuestas correctas posterior a la intervención.
En ambas comunas, cerca del $90 \%$ de la población reconoció la retina como la principal estructura ocular afectada por el parásito.

Acerca del diagnóstico, el 69,2\% (9/13) en la comuna 6 reconoce una prueba de anticuerpos como el método diagnóstico inicial para la infección. 
El conocimiento del tratamiento de la enfermedad mejoró notablemente en la comuna 6 , pasando del 53,8 \% (7/13) al 91,7 \% (11/12) de preguntas correctas.

Todas las personas encuestadas en ambas comunas señalaron que el agua hervida constituye un factor protector contra la enfermedad, y en caso de enfermarse, todos acudirían a un especialista para tratarse.

Respecto al diagnóstico, paradójicamente se presentó un mayor porcentaje de respuestas correctas en la encuesta preintervención. Esto puede explicarse por la especificidad de la pregunta en la encuesta posterior a la intervención educativa, donde se preguntaba a los participantes, con mayor precisión, el tipo de prueba diagnóstica (por ejemplo: anticuerpos, partes del parásito, células del cuerpo, hormonas) que se utilizaba para diagnosticar la toxoplasmosis.

\section{Discusión}

Antes de la intervención, el conocimiento de la población de estudio respecto a la toxoplasmosis era limitado y variaba según los diferentes aspectos de la enfermedad. Cerca de la mitad de los encuestados consideraban que la enfermedad era transmitida por una bacteria; sin embargo, posterior a la intervención, se evidenció una mejoría en el porcentaje de respuestas correctas y se identificó al parásito Toxoplasma gondii como el agente causal.

En un estudio desarrollado en mujeres gestantes y no gestantes en un centro médico en Cuba, se encontraron conocimientos no satisfactorios en cuanto a las vías de transmisión, las medidas de prevención, los factores de exposición y las manifestaciones clínicas de la toxoplasmosis [37]. En contraste, nuestro estudio identificó un mayor conocimiento de los síntomas en mujeres que en hombres, similar a los resultados hallados en estudiantes de una Facultad de Ciencias Veterinarias en la Ciudad de Esperanza, Argentina [38]. Es probable que las mujeres, debido a las dinámicas sociales, estén expuestas con mayor frecuencia a sesiones de educación en salud, y las mujeres gestantes reciben mayor educación en estos temas en los controles prenatales.

En el estudio argentino, el porcentaje de respuestas correctas respecto a la transmisión de la enfermedad fue del 55,5\% [38], siendo este mayor que el de nuestra población de estudio (34,6\%). Sin embargo, posterior a la intervención educativa, este porcentaje aumentó de manera importante en los encuestados tanto de la comuna 1 $(76,9 \%)$ como para la comuna $6(61,5 \%)$. De esa población de estudio, es de anotar que el 77,7 \% consume la carne bien cocida, similar al 78,6 \% de los encuestados de la comuna 6 , que dice cocinar bien la carne antes del consumo, pero inferior al 92,3\% de la población de la comuna 1 que tiene dicha práctica.
El consumo de agua cruda se evidenció en el 22,2\% de la población de estudio, siendo menor a lo reportado en un estudio que evaluó los CAP sobre infecciones de transmisión vertical en un hospital del municipio de Ciénaga, Magdalena, donde el consumo de agua de ríos y quebradas directamente fue del $29 \%$ de las encuestadas [39], y menor a los resultados encontrados por nuestro grupo previamente, donde en casos de toxoplasmosis gestacional, el 71,4 \% de los casos y el $50 \%$ de los controles consumían agua sin hervir [40], atribuyendo de esta forma que la adquisición de la infección podría ser la consecuencia de tomar agua de fuentes no potables como ríos, quebradas, pozos, entre otras.

En Durango, México, en una población de amas de casa, los conocimientos acerca de la enfermedad fueron pobres, al igual que las prácticas para prevenir la infección. Menos del $10 \%$ de las encuestadas conocían algo sobre el parásito, la enfermedad o cómo ocurre la infección y los síntomas que genera, y el 13,5 \% consumía agua sin tratar [29]. En cuanto al hospedero definitivo de la infección, el $20 \%$ acertó en el gato como hospedero, siendo una menor proporción que en el presente estudio.

Adicionalmente, de una población de mujeres embarazadas encuestadas en Estados Unidos por el Colegio Americano de Ginecólogos y Obstetras [30], el $61 \%$ respondió que "el organismo se elimina por las heces de los gatos", siendo inferior al conocimiento del hospedero que presentó nuestra población; y solo el $30 \%$ de las embarazadas sabía que el parásito se puede encontrar en la carne cruda o poco cocida, siendo este porcentaje mayor al hallado en nuestro trabajo, donde el 26,9 \% pensaba que la carne era la principal forma de transmisión. A pesar de lo anterior, el $92 \%$ (comuna 1) y el $78 \%$ (comuna 6) de los encuestados manifestaban cocer la carne por completo, lo que podría disminuir el riesgo de infectarse en nuestra población.

Llama la atención que en dicho estudio [31] no señalan el consumo de agua cruda o no tratada como fuente importante de transmisión, siendo reportado por varios estudios ecoepidemiológicos, en donde se han detectado tanto quistes como ADN del parásito en agua potable $[22,23]$ y donde se ha asociado el consumo de agua cruda a la presencia de la enfermedad [40-45]. Sumado a esto, existe evidencia de brotes de toxoplasmosis provenientes de fuentes de agua en varias comunidades alrededor del mundo [43,46-47].

En la región del eje cafetero, se ha encontrado ADN de Toxoplasma gondii en muestras de agua y carne $[22,48]$; por este motivo, es de suma importancia promover en la comunidad actividades que lleven a la prevención de la infección asociada a dichas fuentes.

En la mayoría de las preguntas relacionadas con conocimientos y actitudes, los participantes mejoraron notablemente en el porcentaje de respuestas correctas 
luego de la intervención; sin embargo, se debe insistir en aspectos generales y específicos del diagnóstico y el tratamiento de la enfermedad. Es de importancia desarrollar actividades subsecuentes sobre los principales aspectos de la enfermedad, para evaluar el impacto de la intervención a largo plazo.

Este estudio es el primero en evaluar los CAP de una población de la ciudad de Armenia, Quindío, acerca de la toxoplasmosis, y permitirá desarrollar estrategias de promoción de la salud y prevención de la enfermedad, en particular, de esta enfermedad infecciosa que es altamente prevalente en la población de la región. Nuestra estrategia educativa fue efectiva en cuanto a la mejoría en el porcentaje de respuestas correctas en la mayoría de los tópicos de conocimientos y en los comportamientos en la encuesta preintervención versus la postintervención.

En una revisión sistemática del impacto de las estrategias de educación en salud sobre el CAP de seroconversión de Toxoplasma en el embarazo [49], se evaluaron 6 estudios de Europa y Canadá, donde su mayoría fueron estudios de casos y controles, y trataron de establecer la relación entre diferentes herramientas comunicativas a largo plazo y la disminución de la seroprevalencia, sugiriendo que los enfoques de educación para la salud pueden ayudar a prevenir la toxoplasmosis congénita. Tres de los cuatro estudios revisados revelaron asociaciones claras de conocimiento y comportamiento con variables demográficas como edad, embarazo previo, lugar de residencia, profesión y nivel educativo. Dos estudios señalaron varias asociaciones de un mayor conocimiento sobre la toxoplasmosis congénita y un "comportamiento más saludable en cuanto a las medidas de prevención de la enfermedad (higiene personal, higiene de los alimentos, higiene de las mascotas), en personas con un mayor nivel educativo y una profesión en el campo médico" [48]; sin embargo, algunos autores afirman que se requieren estudios controlados aleatorizados de educación en salud [48], utilizando técnicas de educación actualizadas, con el fin de mejorar las fuentes y calidad de la información suministrada.

Nuestras limitaciones en el tamaño de la muestra se explican en parte por las medidas de distanciamiento social iniciadas a causa de la emergencia sanitaria decretada por la introducción de la enfermedad por el nuevo coronavirus 2019 (COvid-19) en Colombia en marzo de $2020 \mathrm{y}$, por lo tanto, no nos permite hacer inferencias a toda la población, dada la naturaleza descriptiva del mismo.

Sin embargo, este estudio describe, por primera vez en Colombia, el nivel de los CAP sobre la toxoplasmosis humana en población general. Mostramos que una estrategia educativa basada en el conocimiento claro y preciso de factores influyentes en la exposición al parásito Toxoplasma gondii puede mejorar los CAP respecto al riesgo de adquirir la enfermedad en una población con alta exposición a esta infección.

\section{Agradecimientos}

Este trabajo fue posible gracias al financiamiento del Ministerio de Ciencia Tecnología e Innovación. y la Vicerrectoría de Investigaciones de la Universidad del Quindío. Daniel Celis Giraldo es Joven Investigador, apoyado por MinCiencias, mediante el contrato CT 7732018.

Agradecemos al equipo de apoyo del programa de Comunicación Social y Periodismo, a la Oficina de Apropiación Social del Conocimiento liderada por el profesor Alejandro Herrera y a Johana Burbano.

\section{Declaración de fuente de financiación}

Ministerio de Ciencia, Tecnología e Innovación (Minciencias), contrato 773 de 2018 y Vicerrectoría de Investigaciones de la Universidad del Quindío.

\section{Declaración de conflictos de intereses}

Los autores declaramos no tener ningún conflicto de interés.

\section{Declaración de responsabilidad}

Declaramos que los puntos de vista expresados en este trabajo son responsabilidad exclusiva de los autores y no de las instituciones de las que hacemos parte o de la fuente de financiación.

\section{Declaración de contribución por auto-} res

Todos los autores participaron en la concepción y el diseño del estudio, además de en la recopilación de los datos. Daniel Celis Giraldo y Jorge Enrique Gómez-Marín realizaron el análisis de los datos y participaron en la redacción del artículo.

Laura Lorena García López, Juan David Valencia Hernández, John Alejandro Acosta Dávila y Mónica Vargas Montes participaron en la revisión crítica del artículo y en la aprobación de la versión final del mismo. Los autores se responsabilizan de todos los aspectos del trabajo. 


\section{Referencias}

1. Gómez-Marín JE. Protozoologia medica. Protozoos parasitos en el contexto latinoamericano. Bogotá: Manual Moderno; 2010.

2. Dubey JP. History of the discovery of the life cycle of Toxoplasma gondii. Int J Parasitol. 2009;39(8):877-82. DoI: https://doi. org/10.1016/j.ijpara.2009.01.005

3. Saadatnia G, Golkar M. A review on human toxoplasmosis. Scand J Infect Dis. 2012;44(11):805-14. DoI: https://doi.org/10.3109/00 365548.2012 .693197

4. Zamora-Vélez A, Triviño J, Cuadrado-Ríos S, et al. Detection and genotypes of Toxoplasma gondii DNA in feces of domestic cats in Colombia. Parasite. 2020;27:25. DoI: https://doi.org/10.1051/ parasite/2020023

5. Montazeri M, Mikaeili Galeh T, Moosazadeh M, et al. The global serological prevalence of Toxoplasma gondii in felids during the last five decades (1967-2017): A systematic review and meta-analysis. Parasit Vectors. 2020;13(1):82. DoI: https://doi. org/10.1186/s13071-020-3954-1

6. Dubey JP, Gomez-Marin JE, Bedoya A, et al. Genetic and biologic characteristics of Toxoplasma gondii isolates in free-range chickens from Colombia, South America. Vet Parasitol. 2005;134(12):67-72. DOI: https://doi.org/10.1016/j.vetpar.2005.07.013

7. Wallon M, Peyron F. Congenital toxoplasmosis: A plea for a neglected disease. Pathogens. 2018;7(1):25. DoI: https://doi. org/10.3390/pathogens 7010025

8. Moncada PA, Montoya JG. Toxoplasmosis in the fetus and newborn: An update on prevalence, diagnosis and treatment. Expert Rev Anti Infect Ther. 2012;10(7):815-28. DoI: https://doi. org/10.1586/eri.12.58

9. Gómez-Marin JE, De-la-Torre A, Angel-Muller E, et al. First colombian multicentric newborn screening for congenital toxoplasmosis. Cappello M, editor. PLoS Negl Trop Dis. 2011;5(5):e1195. DOI: https://doi.org/10.1371/journal.pntd.0001195

10. De-la-Torre A, López-Castillo CA, Rueda JC. Clinical patterns of uveitis in two ophthalmology centres in Bogota, Colombia. Clin Experiment Ophthalmol. 2009;37(5):458-66. DOI: https://doi. org/10.1111/j.1442-9071.2009.02082.x

11. Montoya J, Liesenfeld O. Toxoplasmosis. Lancet. 2004;363(9425):1965-76. DoI: https://doi.org/10.1016/S01406736(04)16412-X

12. Brydak-Godowska J, Borkowski PK, Rabczenko D, et al. Do pregnancy, postpartum period and lactation predispose to recurrent toxoplasmic retinochoroiditis? Med Sci Monit. 2015;21:5824. DOI: https://doi.org/10.12659/MSM.892220. PMID: 25703198; PMCID: PMC4349134.

13. Cañón-Franco WA, López-Orozco N, et al. An overview of seventy years of research (1944-2014) on toxoplasmosis in Colombia, South America. Parasit Vectors. 20144;7:427. DoI: https://doi. org/10.1186/1756-3305-7-427

14. Villena I, Ancelle T, Delmas C, et al. Congenital toxoplasmosis in France in 2007: First results from a national surveillance system. Eurosurveillance. 2010;15(25):1-6. DoI: https://doi.org/10.2807/ ese.15.25.19600-en

15. Pleyer U, Groß U, Schlüter D, et al. Toxoplasmosis in Germany: Epidemiology, diagnosis, risk factors, and treatment. Dtsch Arztebl Int. 2019. Dor: https://doi.org/10.3238/arztebl.2019.0435

16. Alvarez C, De-la-Torre A, Vargas M, et al. Striking divergence in Toxoplasma ROP16 nucleotide sequences from human and meat samples. J Infect Dis. 2015;211(12):2006-13. DoI: https://doi. org/10.1093/infdis/jiu833
17. De-la-Torre A, Sauer A, Pfaff AW, et al. Severe South American ocular toxoplasmosis is associated with decreased Ifn- $\gamma / \mathrm{Il}-17 \mathrm{a}$ and increased I1-6/I1-13 intraocular levels. Jardim A, editor. PLoS Negl Trop Dis. 2013;7(11):e2541. DoI: https://doi.org/10.1371/ journal.pntd.0002541

18. Bottos J, Miller RH, Belfort RN, et al. Bilateral retinochoroiditis caused by an atypical strain of Toxoplasma gondii. Br J Ophthalmol. 2009;93(11):1546-50. DOI: http://dx.doi.org/10.1136/ bjo.2009.162412

19. De-la-Torre A, López-Castillo CA, Gómez-Marín JE. Incidence and clinical characteristics in a Colombian cohort of ocular toxoplasmosis. Eye. 2009;23(5):1090-3. DoI: https://doi.org/10.1038/ eye.2008.219

20. Cañón-Franco W, López-Orozco N, et al. An overview of seventy years of research (1944-2014) on toxoplasmosis in Colombia, South America. Parasit Vectors. 2014;7(1):427. DoI: https://doi. org/10.1186/1756-3305-7-427

21. De-la-Torre A, González G, et al. Screening by ophthalmoscopy for Toxoplasma retinochoroiditis in Colombia. Am J Ophthalmol. 2007;143(2):354-6. DoI: https://doi.org/10.1016/j. ajo.2006.09.048

22. Lora-Suarez F, Rivera R, et al. Detection of protozoa in water samples by formalin/ether concentration method. Water Res. 2016;100:377-81. DOI: https://doi.org/10.1016/j.watres.2016.05.038

23. Triviño-Valencia J, Lora F, et al. Detection by PCR of pathogenic protozoa in raw and drinkable water samples in Colombia. Parasitol Res. 2016;115(5):1789-97. DoI: https://doi.org/10.1007/ s00436-016-4917-5

24. Luna JC, Zamora A, Hernández-Arango N, et al. Food safety assessment and risk for toxoplasmosis in school restaurants in Armenia, Colombia. Parasitol Res [internet]. 2019;118(12):3449-57. DOI: https://doi.org/10.1007/s00436-019-06473-w

25. De-La-Torre A, González-López G, Montoya-Gutiérrez JM, et al. Quality of life assessment in ocular toxoplasmosis in a Colombian population. Ocul Immunol Inflamm. 2011;19(4):262-6. DOI: https://doi.org/10.3109/09273948.2011.582220

26. Peyron F, Garweg JG, Wallon M, et al. Long-term impact of treated congenital toxoplasmosis on quality of life and visual performance. Pediatr Infect Dis J. 2011;30(7):597-600. DoI: https://doi. org/10.1097/INF.0b013e31820bb5f3

27. Gómez-Marín JE, Muñoz-Ortiz J, et al. High frequency of ocular toxoplasmosis in Quindío, Colombia and risk factors related to the infection. Heliyon [internet]. 2021;7(4):e06659. DoI: https://doi. org/10.1016/j.heliyon.2021.e06659

28. Organización Panamericana de la Salud. Educación para la salud con enfoque integral. Organ Panam la Salud [internet]. 2014;3. Disponible en: https://www.paho.org/hq/dmdocuments/2017/promocion-salud-intersectorialidad-concurso-2017-educacion.pdf

29. Velázquez-Hernández N, Avilés Ávila AY, Rivas-González MA, et al. Knowledge and practices regarding toxoplasmosis in housewives: A cross sectional study in a northern Mexican city. Dou Z, editor. PLoS One. 2019;14(9):e0222094. DoI: https://doi. org/10.1371/journal.pone.0222094

30. Jones JL, Ogunmodede F, Scheftel J, et al. Toxoplasmosis-Related knowledge and practices among pregnant women in the United States. Infect Dis Obstet Gynecol. 2003;11(3):139-45. DOI: https://doi.org/10.1080/10647440300025512

31. Carter AO, Gelmon SB, et al. The effectiveness of a prenatal education programme for the prevention of congenital toxoplasmosis. Epidemiol Infect. 1989;103(3):539-45. Dor: https://doi. org/10.1017/s0950268800030934 
32. Pawlowski ZS, Gromadecka-Sutkiewicz M, Skommer J, et al. Impact of health education on knowledge and prevention behavior for congenital toxoplasmosis: The experience in Poznań, Poland. Health Educ Res. 2001;16(4):493-502. DoI: https://doi. org/10.1093/her/16.4.493

33. Hernández V. Estudios epidemiológicos: tipos, diseño e interpretación. Enferm inflam intest dia. 2017;16(3):98-105. DoI: https:// doi.org/10.1016/j.eii.2017.03.001

34. Sistema de Información Geográfica del Quindío. [Mapa iteractivo de Quindío]. [citado 2021 mar. 1]. Disponible en: http://190.255.42.26/visor/

35. World Medical Association. World Medical Association Declaration of Helsinki: Ethical principles for medical research involving human subjects. JAMA. 2013;310(20):2191-4. DOI: https://doi org/10.1001/jama.2013.281053

36. Mateus JC, Varela MT, Caicedo DM, Arias NL, Jaramillo CD, Morales LC, et al. ¿Responde la Resolución 8430 de 1993 a las necesidades actuales de la ética de la investigación en salud con seres humanos en Colombia? Biomédica [internet]. 2019 [citado 2021 mar. 1]; 39(3):448-63. Disponible en: https://revistabiomedica.org/index.php/biomedica/article/view/4333

37. Hernández. NP, Giorino E, Álvarez D, et al. Conocimientos sobre toxoplasmosis de las mujeres en edad fértil de un consultorio médico en Trinidad, Sancti Spiritus. Rev Cubana Med Gen Integr. [internet]. 2014 [citado 2021 mar. 1]; 30(2):217-24. Disponible en: http://scielo.sld.cu/scielo.php?script=sci_arttext\&pid=S086421252014000200007\&lng=es

38. Monti M, Marti M, Sequeira S, et al. Evaluación del conocimiento sobre toxoplasmosis, de la población de la ciudad de Esperanza, República Argentina. Rev. FAVE - Ciencias Veterianarias [internet]. 2002 [citado 2021 mar. 1]; 1(2):1-13. Disponible en: https:// bibliotecavirtual.unl.edu.ar/publicaciones/index.php/FAVEveterinaria/article/view/1375.

39. Ariza K, Atencio Yepez M, et al. Conocimientos, actitudes y prácticas de las infecciones de transmisión vertical, en mujeres embarazadas atendidas en el Hospital San Cristóbal de Ciénaga. Duazary [internet] 2013 [citado 2021 mar. 1]; 8(1):42-7. Disponible en: https://revistas.unimagdalena.edu.co/index.php/duazary/ article/view/250

40. López-Castillo CA, Díaz-Ramírez J, Gómez-Marín JE. Factores de riesgo en mujeres embarazadas, infectadas por Toxoplasma gondii en Armenia-Colombia. Rev Salud Pública [internet].
2005;7(2):180-90. Disponible en: https://www.scielosp.org/pdf/ rsap/2005.v7n2/180-190/es

41. Shapiro K, Bahia-Oliveira L, Dixon B, Dumètre A, de Wit LA, VanWormer E, et al. Environmental transmission of Toxoplasma gondii: Oocysts in water, soil and food. Food Waterborne Parasitol. 2019;15:e00049. Dor: https://doi.org/10.1016/j.fawpar.2019. e00049

42. Bahia-Oliveira LMG, Jones JL, Azevedo-Silva J, et al. Highly endemic, waterborne toxoplasmosis in North Rio de Janeiro State, Brazil. Emerg Infect Dis. 2003;9(1):55-62. DoI: https://doi. org/10.3201/eid0901.020160

43. De Moura L, Bahia-Oliveira LMG, Wada MY, et al. Waterborne toxoplasmosis, Brazil, from field to gene. Emerg Infect Dis [internet]. 2006;12(2):326-9. DOI: https://doi.org/10.3201/ eid1202.041115

44. Vieira FP, Alves MDG, Martins LM, et al. Waterborne toxoplasmosis investigated and analysed under hydrogeological assessment: New data and perspectives for further research. Mem Inst Oswaldo Cruz [internet]. 2015;110(7):929-35. DoI: https:// doi.org/10.1590/0074-02760150262

45. Bowie WR, King AS, Werker DH, et al. Outbrak of toxoplasmosis associated with municipal drinking water. Lancet. 1997;350(9072):173-7. DOI: https://doi.org/10.1016/S01406736(96)11105-3

46. Jones JL, Akstein RB, Hlavsa MC, et al. Follow-up of the 1977 Georgia outbreak of toxoplasmosis. Am J Trop Med Hyg. 2016; 94(6):1299-300. DoI: https://doi.org/10.4269/ajtmh.15-0919

47. Palanisamy M, Madhavan B, Balasundaram M, et al. Outbreak of ocular toxoplasmosis in Coimbatore, India. Indian J Ophthalmol. 2006;54(2):129-131. DOI: https://doi.org/10.4103/03014738.25839

48. Lora-Suárez F, Perez JE, Arias LE, et al. Detección de Toxoplasma gondii en carnes de consumo humano por la técnica de reacción en cadena de la polimerasa en tres ciudades del eje cafetero. Infectio [internet]. 2007 [citado 2021 mar. 1]; 1(3):117-23. Disponible en: https://www.revistainfectio.org/index.php/infectio/ article/view/150/185

49. Di Mario S, Basevi V, Gagliotti C, et al. Prenatal education for congenital toxoplasmosis. Cochrane Database Syst Rev. [internet]. 2013 [citado 2021 mar. 1]; (2):CD006171. https://pubmed. ncbi.nlm.nih.gov/23450566/. Update in: Cochrane Database Syst Rev. 2015;(10):CD006171. PMID: 23450566 